\title{
Comparison of MMP2 and MMP9 expression levels between primary and metastatic regions of oral squamous cell carcinoma
}

\author{
Kensuke Nishio'), Keiko Motozawa2), Daisuke Omagari3), Takahiro Gojoubori3), \\ Takayuki Ikeda4), Masatake Asano ${ }^{3)}$, and Nobuhito Gionhaku ${ }^{4}$ \\ 1)Division of Applied Oral Sciences, Nihon University Graduate School of Dentistry, Tokyo, Japan \\ 2)Division of Oral Structural and Functional Biology, Nihon University Graduate School of Dentistry, \\ Tokyo, Japan \\ 3)Department of Pathology, Nihon University School of Dentistry, Tokyo, Japan \\ 4)Department of Complete Denture Prosthodontics, Nihon University School of Dentistry, Tokyo, Japan
}

(Received October 9, 2015; Accepted December 1, 2015)

\begin{abstract}
Matrix metalloproteinases (MMPs) and tumor-associated macrophages (TAMs) play important roles in tumor growth. The present study investigated the expression levels of MMP2 and MMP9 in relation to the distribution of TAMs in the primary and metastatic regions of oral squamous cell carcinoma. Twenty-nine cases of oral squamous cell carcinoma (OSCC) with regional lymph node metastasis were selected from available documents in the archives of the Department of Pathology, Nihon University School of Dentistry. Four-micrometerthick sections were prepared from the primary and metastatic regions. Each section was subjected to immunohistochemical staining using anti-MMP2, anti-MMP9, and anti-CD68 antibodies. The distribution and localization of MMPs and TAMs were compared between primary and metastatic regions. The expression levels of both MMPs were higher in the metastatic regions of lingual and gingival cancers. Statistically significant differences were observed in both $\mathrm{T} 1$ and $\mathrm{T} 2$ cases. In contrast to the higher expression of MMPs in metastatic regions, a higher number of TAMs were distributed in the primary regions. From these results, MMP expression levels
\end{abstract}

Correspondence to Dr. Masatake Asano, Department of Pathology, Nihon University School of Dentistry, 1-8-13 Kanda-Surugadai, Chiyoda-ku, Tokyo, 101-8310, Japan

Fax: +81-3-3219-8340 E-mail: asano.masatake@nihon-u.ac.jp

doi.org/10.2334/josnusd.58.59

DN/JST.JSTAGE/josnusd/58.59 and the numbers of TAMs were expected to have an inverse relationship between the primary and metastatic regions of OSCC. (J Oral Sci 58, 59-65, 2016)

Keywords: oral squamous cell carcinoma (OSCC); matrix metalloproteinases (MMPs); tumorassociated macrophages (TAMs).

\section{Introduction}

The complex interplay between tumor cells and their microenvironment plays a pivotal role in carcinogenesis and cancer progression (1). Matrix metalloproteinases (MMPs) are the most important participants of extracellular matrix remodeling $(1,2)$. They belong to the zinc-dependent family of endopeptidases which are implicated in a variety of physiological processes $(2,3)$. One of the most relevant functions of MMPs is the degradation of physical barriers such as the basement membrane (4-7). As the metastatic potential of various cancers depends on the ability of tumor cells to degrade the basement membrane, MMPs are the main targets of therapeutic agents (3). Among them, MMP2 and MMP9 have the ability to cleave type IV collagen, the main component of the basement membrane and to contribute to the metastasis of tumor cells (4-7).

Tumor-associated macrophages (TAMs) have various effects on tumor growth (5-8). Although the existence of TAMs in the metastatic region has been recognized, the role of these cells has only recently been appreciated.

Oral squamous cell carcinoma (OSCC) has the highest 
incidence rates among malignant tumors of the head and neck (9) and is one of the six most common cancers. Although the expression of MMPs and distribution of TAMs in OSCC have been intensely studied $(8,10,11)$, a comparison of the expression levels and distribution patterns in the primary and metastatic regions has not been well defined. Research in these fields will help provide insight into the clinical behavior of OSCC. Thus, the aim of this study was to compare the expression levels of MMP2 and MMP9 in relation to TAM distribution in the primary and metastatic regions of OSCC.

\section{Materials and Methods}

\section{Case selection}

Twenty-nine OSCC cases with regional lymph node metastasis were chosen for this study. The surgical specimens were obtained from the Dental Hospital of Nihon University School of Dentistry. The histopathological diagnoses of these specimens were independently confirmed by two oral pathologists. Additionally, the differentiation degree (grades I-III) of the tumors was also assessed. The locations of the primary tumors were lingual $(n=13)$, gingival $(n=14)$, and buccal mucosa $(n$ $=2$ ). This study was conducted in accordance with the Declaration of Helsinki with respect to medical protocol and ethics, and the regional Ethical Review Board of Nihon University School of Dentistry approved this study (EP2015-11).

\section{Antibodies}

Anti-MMP2, anti-MMP9, and anti-CD68 antibodies (Abs) were purchased from Abcam (Cambridge, UK), Santa Cruz (CA, USA), and Dako (Tokyo, Japan), respectively. Anti-MMP2 and anti-CD68 Abs were diluted to 1/50 and anti-MMP9 $\mathrm{Ab}$ was diluted to 1/100 using 10 $\mathrm{mM}$ Tris- $\mathrm{HCl}$ ( $\mathrm{pH}$ 7.5)-buffered saline supplemented with $1 \%$ bovine serum albumin (1\% BSA-TBS).

\section{Immunohistochemistry}

All the samples were fixed in $10 \%$ formalin and embedded in paraffin. Four-micrometer-thick sections were prepared, de-paraffinized in xylene, and rehydrated with $100 \%$ ethanol. Endogenous peroxidase activity was inactivated with $0.3 \%$ hydrogen peroxide in methanol for $20 \mathrm{~min}$ at room temperature (RT). The sections were then boiled in $10 \mathrm{mM}$ citrate buffer ( $\mathrm{pH} \mathrm{6.0)}$ for $20 \mathrm{~min}$ and cooled. To block non-specific binding, the sections were incubated with $1 \%$ BSA-TBS for $1 \mathrm{~h}$ at RT. Thereafter, the blocking solution was removed, and the first antibodies were applied and incubated for $1 \mathrm{~h}$ at RT or overnight at $4^{\circ} \mathrm{C}$ (Table 1). Negative control studies were performed with $1 \%$ BSA-TBS instead of the first antibody. The sections were then incubated with horseradish peroxidase (HRP)-conjugated goat anti-mouse IgG or anti-rabbit IgG (diluted to $1 / 500$ ) for $1 \mathrm{~h}$ at RT. After the sections were washed, they were developed with freshly prepared diaminobenzidine chromogen solution (Sigma, Tokyo, Japan) for $7 \mathrm{~min}$, counterstained with hematoxylin for 10 $\mathrm{s}$, dehydrated in a series of ethanol dilutions, cleared in xylene, and mounted on glass coverslips.

\section{Immunohistochemical evaluation}

The sections were evaluated in three different randomly selected fields, each of which contained tumor nests and stroma in approximately equal proportions. For the evaluation of MMP expression levels, the sections were scored by determining the highest score number. The staining intensities were classified into four different levels: 0 , negative; 1 , low; 2 , moderate; and 3 , high. For the CD68-positive TAMs, the sections were scored by determining the average number of stained cells observed under a light microscope using a micrometer (Olympus, Tokyo, Japan) at $200 \times$ magnification. The results have been expressed as mean \pm standard deviation values.

\section{Statistical analysis}

Statistical analysis was performed using a two-tailed Student's $t$-test, and $P$ values less than 0.05 were considered to be significant.

\section{Results}

Table 2 shows the clinical data of the OSCC cases examined in this study. The age of the patients ranged from 18 to 88 years, with a mean age of 62 years. The patients included 17 men and 12 women, at a ratio of 1.4:1. Clinicopathological data were determined according to the guidelines of the Union Internationale Contre le Cancer (UICC) (12). Immunohistochemical staining showed that both MMP2 and MMP9 were present in the cytoplasm of the tumor cells (Fig. 1). The expression levels of the MMPs were compared between the primary and metastatic regions. In lingual cancer, equivalent levels of MMP9 expression were detected in both regions (Fig. 1A, B), and there were no significant differences between the regions (Fig. 1C: $P=0.482$ ). In contrast, 1.7-fold higher MMP9 expression was observed in the metastatic region of gingival cancer (Fig. 1D, E) than in the primary region, but this difference was not statistically significant (Fig. 1F: $P=0.082$ ). In spite of the clear expression of MMP9, MMP2 expression was barely detectable in the primary regions of both lingual and gingival cancer (Fig. 1G, J). Slight MMP2 expres- 
Table 1 Primary antibodies used in this study

\begin{tabular}{lllllll}
\hline Antibody & Host & Clone & Dilution & Antigen retrieval & Incubation & Supplier \\
\hline MMP2 & mouse & CA-400/CA719E3C & $50 \times$ & CB, 98 ${ }^{\circ} \mathrm{C} ; 20 \mathrm{~min}$ & $4^{\circ} \mathrm{C}, 18 \mathrm{~h}$ & Abcam \\
MMP9 & rabbit & C-20 & $100 \times$ & CB, $98^{\circ} \mathrm{C} ; 20 \mathrm{~min}$ & $\mathrm{RT}, 1 \mathrm{~h}$ & Santa Cruz \\
CD68 & mouse & PG-M1 & $50 \times$ & $\mathrm{CB}, 98^{\circ} \mathrm{C} ; 20 \mathrm{~min}$ & $\mathrm{RT}, 1 \mathrm{~h}$ & Dako \\
\hline
\end{tabular}

MMP, matrix metalloproteinase; $\mathrm{CB}$, citrate buffer; RT, room temperature

Table 2 Clinical classification of 29 oral squamous cell carcinoma cases

\begin{tabular}{|c|c|c|c|c|c|c|c|c|c|}
\hline \multirow{2}{*}{ No. } & \multirow{2}{*}{ age } & \multirow{2}{*}{$\operatorname{sex}$} & \multirow{2}{*}{ primary site } & \multicolumn{2}{|c|}{ TNM classification } & \multirow{2}{*}{ M } & \multirow{2}{*}{ Class of UICC } & \multirow{2}{*}{ Grade } & \multirow{2}{*}{$\begin{array}{l}\text { Duration for } \\
\text { metastasis (day) }\end{array}$} \\
\hline & & & & $\mathrm{T}$ & $\mathrm{N}$ & & & & \\
\hline 1 & 51 & M & lingual & 2 & $2 b$ & 0 & IVA & II & 248 \\
\hline 2 & 61 & M & lingual & 2 & $2 b$ & 0 & IVA & I & 103 \\
\hline 3 & 67 & M & lingual & 1 & 1 & 0 & III & I & 412 \\
\hline 4 & 46 & M & lingual & 2 & $2 b$ & 0 & IVA & II & 273 \\
\hline 5 & 70 & M & lingual & 3 & 1 & 0 & III & I & 109 \\
\hline 6 & 81 & $\mathrm{~F}$ & lingual & 2 & 1 & 0 & III & I & 371 \\
\hline 7 & 59 & M & lingual & 2 & $2 \mathrm{a}$ & 0 & IVA & I & 167 \\
\hline 8 & 57 & M & lingual & 2 & $2 b$ & 0 & IVA & I & 231 \\
\hline 9 & 51 & $\mathrm{~F}$ & lingual & 1 & 1 & 0 & III & I & 55 \\
\hline 10 & 66 & M & lingual & 1 & 1 & 0 & III & I & 148 \\
\hline 11 & 52 & M & lingual & 4 & $2 \mathrm{c}$ & 0 & IVA & III & 41 \\
\hline 12 & 64 & $\mathrm{~F}$ & lingual & 3 & $2 \mathrm{c}$ & 0 & IVA & I & 417 \\
\hline 13 & 78 & $\mathrm{~F}$ & lingual & 3 & $2 \mathrm{a}$ & 0 & IVA & I & 27 \\
\hline 14 & 60 & $\mathrm{~F}$ & gingival & 2 & $2 b$ & 0 & IVA & II & 32 \\
\hline 15 & 18 & M & gingival & 2 & $2 b$ & 0 & IVA & II & 166 \\
\hline 16 & 88 & M & gingival & 2 & 1 & 0 & III & I & 210 \\
\hline 17 & 65 & $\mathrm{~F}$ & gingival & & $2 b$ & 0 & & II & 190 \\
\hline 18 & 87 & $\mathrm{~F}$ & gingival & 2 & $2 b$ & 0 & IVA & II & 28 \\
\hline 19 & 69 & M & gingival & 2 & 1 & 0 & III & I & 35 \\
\hline 20 & 52 & M & gingival & 3 & 1 & 0 & III & I & 48 \\
\hline 21 & 67 & $\mathrm{~F}$ & gingival & 2 & $2 b$ & 0 & IVA & I & 19 \\
\hline 22 & 74 & M & gingival & & 1 & 0 & & I & 206 \\
\hline 23 & & M & gingival & & $2 b$ & 0 & & I & 28 \\
\hline 24 & 57 & $\mathrm{~F}$ & gingival & 2 & $2 b$ & 0 & IVA & I & 9 \\
\hline 25 & 60 & $\mathrm{~F}$ & gingival & & $2 b$ & 0 & & I & 146 \\
\hline 26 & 55 & M & gingival & 4 & $2 b$ & 0 & IVA & III & 59 \\
\hline 27 & 65 & M & gingival & 2 & $2 b$ & 0 & IVA & II & 27 \\
\hline 28 & 66 & $\mathrm{~F}$ & buccal & 2 & 1 & 0 & III & II & 37 \\
\hline 29 & 58 & $\mathrm{~F}$ & buccal & 1 & 1 & 0 & III & I & 20 \\
\hline
\end{tabular}

UICC, Union Internationale Contre le Cancer

sion was observed in the metastatic regions (Fig. 1H, K), and a statistically significant difference was observed in lingual cancer (Fig. 1I: $P=0.039$ ) but not in gingival cancer (Fig. 1L: $P=0.111$ ).

The expression levels of the MMPs in the metastatic regions were further compared between lingual and gingival cancers (Fig. 2A, B). MMP9 expression was found to be almost the same in both types, whereas the expression level of MMP2 was slightly higher in the metastatic region of gingival cancer (1.58-fold). However, these differences were not statistically significant for MMP2 and MMP9 ( $P=0.588$ and 0.603 , respectively).

MMP expression levels were analyzed in relation to the clinical data (Table 2). Increased expression of MMP2 ( $P$
$=0.046)$ and MMP9 $(P=0.016)$ was evident in grade $\mathrm{I}$ cases. However, no other clinical data correlated with the MMP expression level.

\section{CD68 labeling index}

The distribution patterns of CD68-positive TAMs were compared between the primary and metastatic regions. TAMs were detected in the stroma of the primary and metastatic regions and were localized not only in the invasive front but also in the non-invasive area (Fig. 3A). The total number of TAMs was counted in lingual and gingival cancers and compared, the results of which are shown in Fig. 3B and C. The highest number of TAMs was detected in the primary regions of both cancers (Fig. 

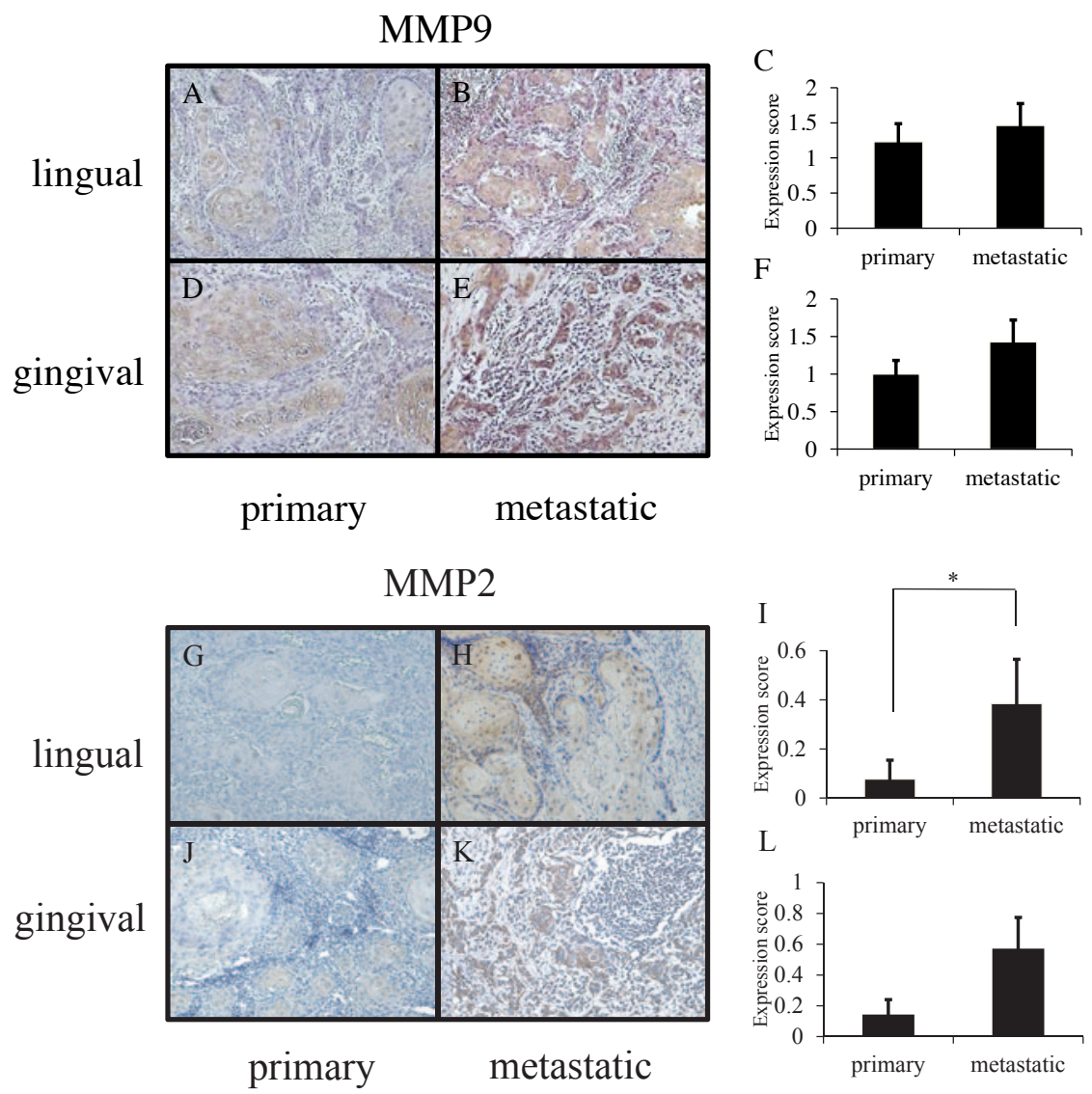

Fig. 1 Specimens from the primary (A, D, G, J) and metastatic regions (B, E, H, K) of OSCC samples were stained with anti-MMP9 (upper panel) and anti-MMP2 (lower panel) Abs. The staining intensities were calculated and compared (C, F, I, L). ${ }^{*} P<0.05$ MMP, matrix metalloproteinase; OSCC, oral squamous cell carcinoma
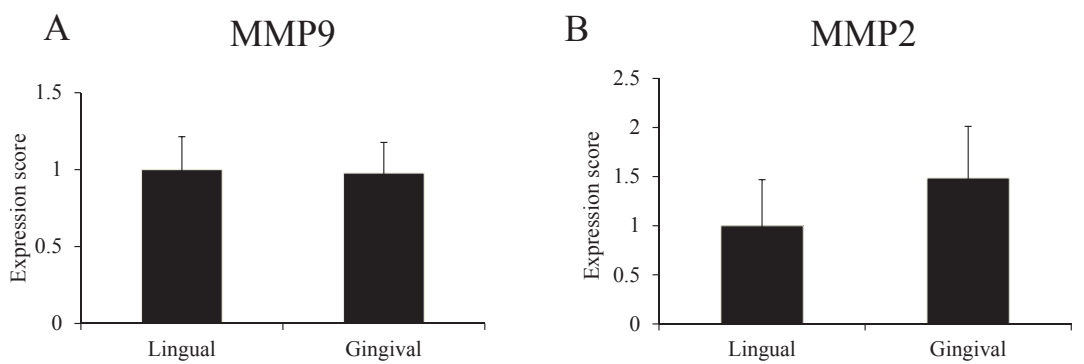

Fig. 2 The MMP9 (A) and MMP2 (B) staining intensities were compared between lingual and gingival cancer. MMP, matrix metalloproteinase

$3 \mathrm{~B}, \mathrm{C})$. When the number in the metastatic region was set as 1 , the number of TAMs in the primary regions reached $1.9(P=0.006)$ and $1.5(P=0.013)$ for lingual and gingival cancers, respectively. The number of CD68-positive cells in the primary regions was analyzed in relation to the clinical data, and a slight correlation was observed between the number of TAMs and the time intervals to neck dissection (Fig. 4).

\section{Discussion}

More than 20 members of the MMP family have been characterized to date (1). Among them, MMP2 and MMP9 belong to the gelatinase family and contribute to the degradation of extracellular substrates such as collagen type IV, the main component of the basement membrane (4,13-15). Many studies have demonstrated the significance of both MMPs in the aggressive growth of OSCCs (1-3). However, there have been no reports 


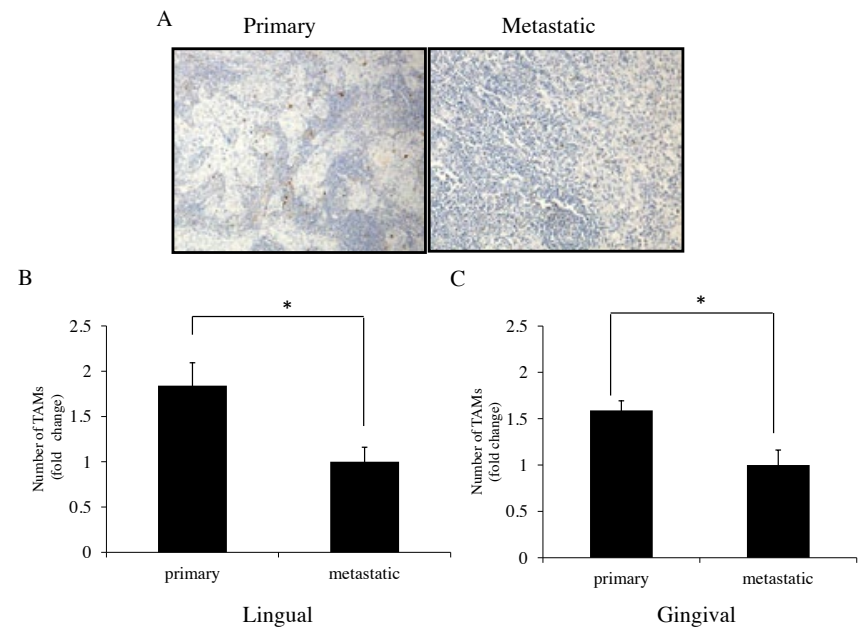

Fig. 3 The distribution of the TAMs in the primary and metastatic regions was compared (A). The number of TAMs was counted and compared between primary and metastatic regions (B and $\mathrm{C}$ ). $* P<0.05$

TAM, tumor-associated macrophages

comparing the expression levels between the primary and the metastatic regions of OSCC. In the present study, we attempted to evaluate the expression profiles of MMP2 and MMP9. Intriguingly, both MMPs were more highly expressed in the metastatic regions than in the primary regions of OSCC, and this pattern was quite prominent in gingival cancers. The increased expression of MMPs correlates with the proliferation and invasion of tumor cells (16-23). The enhancement of MMP activity in cultured cells indicates increased metastatic potential (24-26). It can be speculated that the cancer cells that metastasize to the lymph nodes still retain their metastatic potential and that this metastatic activity is much higher than that of the resident cells in the primary regions. If this is the case, then a question arises regarding factors that control the metastatic activity of cancer cells. One possibility may be the soluble factors secreted by stromal cells or other cell types (27). The susceptible sources for these factors may include TAMs. It has been known for many years that TAMs are the main cellular components of human cancers (5-8). Recent studies have described the development of two distinct subsets of macrophages in response to their tissue environment (7). Macrophages can reversibly change their functions and contribute to both cancer growth and regression. The number of TAMs was higher in the primary region of OSCC, and an inverse correlation was observed between them and MMP expression levels in the present study. The expression of the MMPs is regulated by several factors, including cytokines such as transforming growth factor- $\beta(28,29)$, epidermal growth factor $(30)$, and tumor necrosis factor- $\alpha(31,32)$. Transforming growth factor- $\beta$

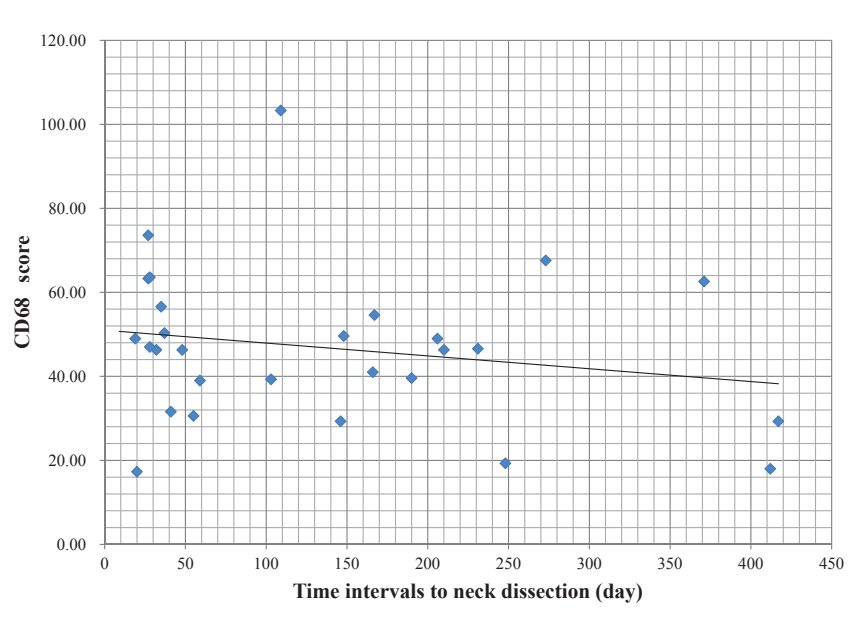

Fig. 4 Relationship between the number of CD68-positive cells and time intervals to neck dissection.

induces the expression of MMP and also simultaneously reduces the expression of collagenase (28). Although the biological meanings of this inverse relationship and the underlying molecular mechanisms remain elusive, TAMs distributed in the primary regions of OSCCs may contribute to the reduction of MMP expression levels through some cytokines.

Most of the regulatory regions of MMP genes contain consensus binding sites for the transcription factor AP-1 (27). Analysis of this region in the MMP2 and MMP9 genes showed that the AP-1 site is only present in the latter gene. In spite of the different composition of the regulatory regions, the expression of both MMPs was low in the primary regions, and this may help explain the AP-1-independent regulatory mechanisms.

Although the MMP expression levels did not correlate with the clinical features of OSCC, a slight correlation was observed between the number of TAMs and the duration of surgery. The number of TAMs in the primary region was associated with the time to surgery. Accumulation of TAMs in the cancer stroma is correlated with angiogenesis in various cancers (33-37), including oral cancer. TAMs also increase the secretion of angiogenic factors such as vascular endothelial growth factor and contribute to the integrity of the vascular structure of OSCC $(8,38)$. However, contradictory data has also been reported indicating a lack of correlation between TAM distribution and angiogenic activity (39). The biological functions of TAMs in OSCC require further investigation.

An inverse relationship was observed between MMP expression intensities and TAM distribution in the 
primary and metastatic regions of OSCC. The results may indicate that the tumor cells in the metastatic region still maintain invasive potential, whereas the TAMs in the primary region may play other roles such as angiogenesis. Further analysis is necessary for a better understanding of OSCC behavior.

\section{Acknowledgments}

This work was supported by the following; Nihon University Joint Research Grant for 2010-2012 (to Dr. Okayama and Dr. Imamura); a grant from the Strategic Research Base Development Program for Private Universities from Ministry of Education, Culture, Sports, Science, and Technology, JAPAN (MEXT), 2010-2014 (S1001024) and 2011-2013 (23592778); Health and Labour Sciences Research Grants and Research on international cooperation in medical science; The Promotion and Mutual Aid Corporation for Private Schools of Japan (2011); a grant-in-aid for Scientific Research (C) (2011-2013).

\section{References}

1. Kessenbrock K, Plaks V, Werb Z (2010) Matrix metalloproteinases: regulators of the tumor microenvironment. Cell 141, 52-67.

2. Page-McCaw A, Ewald AJ, Werb Z (2007) Matrix metalloproteinases and the regulation of tissue remodelling. Nat Rev Mol Cell Biol 8, 221-233.

3. Roy R, Yang J, Moses MA (2009) Matrix metalloproteinases as novel biomarkers and potential therapeutic targets in human cancer. J Clin Oncol 27, 5287-5297.

4. Collier IE, Wilhelm SM, Eisen AZ, Marmer BL, Grant GA, Seltzer JL et al. (1988) H-ras oncogene-transformed human bronchial epithelial cells (TBE-1) secrete a single metalloprotease capable of degrading basement membrane collagen. J Biol Chem 263, 6579-6587.

5. Condeelis J, Pollard JW (2006) Macrophages: obligate partners for tumor cell migration, invasion, and metastasis. Cell 124, 263-266.

6. Lewis CE, Pollard JW (2006) Distinct role of macrophages in different tumor microenvironments. Cancer Res 66, 605-612.

7. Qian BZ, Pollard JW (2010) Macrophage diversity enhances tumor progression and metastasis. Cell 141, 39-51.

8. Li C, Shintani S, Terakado N, Nakashiro K, Hamakawa H (2002) Infiltration of tumor-associated macrophages in human oral squamous cell carcinoma. Oncol Rep 9, 1219-1223.

9. Molinolo AA, Amornphimoltham P, Squarize CH, Castilho RM, Patel V, Gutkind JS (2009) Dysregulated molecular networks in head and neck carcinogenesis. Oral Oncol 45, 324-334.

10. Bôas DS, Takiya CM, Coelho-Sampaio TL, Monção-Ribeiro LC, Ramos EA, Cabral MG et al. (2010) Immunohistochemical detection of Ki-67 is not associated with tumor-infiltrating macrophages and cyclooxygenase- 2 in oral squamous cell carcinoma. J Oral Pathol Med 39, 565-570.

11. El-Rouby DH (2010) Association of macrophages with angio- genesis in oral verrucous and squamous cell carcinomas. J Oral Pathol Med 39, 559-564.

12. Piemonte M (2003) TNM--classification of malignant tumors (VI edition--2002). Innovations in the classification of head and neck neoplasms. Acta Otorhinolaryngol Ital 23, 132-135.

13. Wilhelm SM, Collier IE, Marmer BL, Eisen AZ, Grant GA, Goldberg GI (1989) SV40-transformed human lung fibroblasts secrete a 92-kDa type IV collagenase which is identical to that secreted by normal human macrophages. J Biol Chem 264, 17213-17221.

14. Dumas V, Kanitakis J, Charvat S, Euvrard S, Faure M, Claudy A (1999) Expression of basement membrane antigens and matrix metalloproteinases 2 and 9 in cutaneous basal and squamous cell carcinomas. Anticancer Res 19, 2929-2938.

15. Hagedorn HG, Bachmeier BE, Nerlich AG (2001) Synthesis and degradation of basement membranes and extracellular matrix and their regulation by TGF- $\beta$ in invasive carcinomas (Review). Int J Oncol 18, 669-681.

16. Shima I, Sasaguri Y, Kusukawa J, Yamana H, Fujita H, Kakegawa $\mathrm{T}$ et al. (1992) Production of matrix metalloproteinase-2 and metalloproteinase-3 related to malignant behavior of esophageal carcinoma. A clinicopathologic study. Cancer 70, 2747-2753.

17. Kusukawa J, Sasaguri Y, Shima I, Kameyama T, Morimatsu M (1993) Expression of matrix metalloproteinase-2 related to lymph node metastasis of oral squamous cell carcinoma. A clinicopathologic study. Am J Clin Pathol 99, 18-23.

18. Cazorla M, Hernández L, Nadal A, Balbín M, López JM, Vizoso F et al. (1998) Collagenase-3 expression is associated with advanced local invasion in human squamous cell carcinomas of the larynx. J Pathol 186, 144-150.

19. Sato F, Shimada Y, Watanabe G, Uchida S, Makino T, Imamura M (1999) Expression of vascular endothelial growth factor, matrix metalloproteinase- 9 and E-cadherin in the process of lymph node metastasis in oesophageal cancer. Br J Cancer 80, 1366-1372.

20. Kurahara S, Shinohara M, Ikebe T, Nakamura S, Beppu M, Hiraki A et al. (1999) Expression of MMPS, MT-MMP, and TIMPs in squamous cell carcinoma of the oral cavity: correlations with tumor invasion and metastasis. Head Neck 21, 627-638.

21. Riedel F, Götte K, Schwalb J, Bergler W, Hörmann K (2000) Expression of 92-kDa type-IV collagenase correlates with angiogenic markers and poor survival in head and neck squamous cell carcinoma. Int J Oncol 17, 1099-1105.

22. Ohashi K, Nemoto T, Nakamura K, Nemori R (2000) Increased expression of matrix metalloproteinase 7 and 9 and membrane type 1-matrix metalloproteinase in esophageal squamous cell carcinomas. Cancer 88, 2201-2209.

23. Imanishi $Y$, Fujii M, Tokumaru $Y$, Tomita $T$, Kanke M, Kanzaki J et al. (2000) Clinical significance of expression of membrane type 1 matrix metalloproteinase and matrix metalloproteinase-2 in human head and neck squamous cell carcinoma. Hum Pathol 31, 895-904.

24. Deryugina EI, Soroceanu L, Strongin AY (2002) Up-regula- 
tion of vascular endothelial growth factor by membrane-type 1 matrix metalloproteinase stimulates human glioma xenograft growth and angiogenesis. Cancer Res 62, 580-588.

25. Seiki M (2003) Membrane-type 1 matrix metalloproteinase: a key enzyme for tumor invasion. Cancer Lett 194, 1-11.

26. Yamamoto H, Noura S, Okami J, Uemura M, Takemasa I, Ikeda M et al. (2008) Overexpression of MT1-MMP is insufficient to increase experimental liver metastasis of human colon cancer cells. Int J Mol Med 22, 757-761.

27. Chakraborti S, Mandal M, Das S, Mandal A, Chakraborti T (2003) Regulation of matrix metalloproteinases: an overview. Mol Cell Biochem 253, 269-285.

28. Overall CM, Wrana JL, Sodek J (1991) Transcriptional and post-transcriptional regulation of $72-\mathrm{kDa}$ gelatinase/type IV collagenase by transforming growth factor- $\beta 1$ in human fibroblasts. Comparisons with collagenase and tissue inhibitor of matrix metalloproteinase gene expression. J Biol Chem 266, 14064-14071.

29. Salo T, Lyons JG, Rahemtulla F, Birkedal-Hansen H, Larjava H (1991) Transforming growth factor- $\beta 1$ up-regulates type IV collagenase expression in cultured human keratinocytes. J Biol Chem 266, 11436-11441.

30. Delany AM, Brinckerhoff CE (1992) Post-transcriptional regulation of collagenase and stromelysin gene expression by epidermal growth factor and dexamethasone in cultured human fibroblasts. J Cell Biochem 50, 400-410.

31. Hagemann T, Robinson SC, Schulz M, Trümper L, Balkwill FR, Binder C (2004) Enhanced invasiveness of breast cancer cell lines upon co-cultivation with macrophages is due to TNF- $\alpha$ dependent up-regulation of matrix metalloproteases. Carcinogenesis 25, 1543-1549.
32. Bahar-Shany K, Ravid A, Koren R (2010) Upregulation of MMP-9 production by TNF $\alpha$ in keratinocytes and its attenuation by vitamin D. J Cell Physiol 222, 729-737.

33. Leek RD, Lewis CE, Whitehouse R, Greenall M, Clarke J, Harris AL (1996) Association of macrophage infiltration with angiogenesis and prognosis in invasive breast carcinoma. Cancer Res 56, 4625-4629.

34. Valković T, Dobrila F, Melato M, Sasso F, Rizzardi C, Jonjić $\mathrm{N}$ (2002) Correlation between vascular endothelial growth factor, angiogenesis, and tumor-associated macrophages in invasive ductal breast carcinoma. Virchows Arch 440, 583-588.

35. Hamada I, Kato M, Yamasaki T, Iwabuchi K, Watanabe T, Yamada T et al. (2002) Clinical effects of tumor-associated macrophages and dendritic cells on renal cell carcinoma. Anticancer Res 22, 4281-4284.

36. Chen JJ, Lin YC, Yao PL, Yuan A, Chen HY, Shun CT et al. (2005) Tumor-associated macrophages: the double-edged sword in cancer progression. J Clin Oncol 23, 953-964.

37. Mazibrada J, Rittà M, Mondini M, De Andrea M, Azzimonti B, Borgogna C et al. (2008) Interaction between inflammation and angiogenesis during different stages of cervical carcinogenesis. Gynecol Oncol 108, 112-120.

38. Liu SY, Chang LC, Pan LF, Hung YJ, Lee CH, Shieh YS (2008) Clinicopathologic significance of tumor cell-lined vessel and microenvironment in oral squamous cell carcinoma. Oral Oncol 44, 277-285.

39. Tataroğlu C, Kargi A, Ozkal S, Eșrefoğlu N, Akkoçlu A (2004) Association of macrophages, mast cells and eosinophil leukocytes with angiogenesis and tumor stage in non-small cell lung carcinomas (NSCLC). Lung Cancer 43, 47-54. 